\title{
El problema de la alimentación: un enfoque desde las encuestas de nutrición. Chile, 1928-1938*
}

\section{The Food Problem: An Approach from Nutritional Surveys. Chile, 1928-1938}

\author{
Juan Carlos Yáñez Andrade \\ Universidad de Valparaíso, Valparaíso, Chile; email: juancarlos.yanez@uv.cl
}

\begin{abstract}
Resumen. El presente artículo analiza una serie de encuestas de nutrición entre 1928 y 1938, en el contexto de la preocupación por las condiciones de vida de la clase trabajadora. Estas encuestas, junto con el reconocimiento de la importancia de la alimentación en la estructura de gastos de las familias, permiten identificar patrones de consumo y los serios problemas que las familias más pobres tuvieron para alcanzar un nivel de vida mínimo, medido en términos de consumo de calorías. Además de valorar estas encuestas, este artículo se inserta en un proyecto mayor que busca aportar elementos teóricos y metodológicos para la comprensión del fenómeno de la alimentación y reflexionar históricamente sobre el desarrollo de la ciencia de la nutrición.
\end{abstract}

Palabras clave: alimentación, encuestas de nutrición, políticas de alimentación, Chile.

Abstract. This article analyzes several nutrition surveys conducted between 1928 and 1938 , in the context of the concerns about the living conditions of the working-class. In addition to acknowledging the importance of food in the expenses structure of the working families in the country, these surveys identify consumption patterns and the serious problems that the poor families had to endure in order to attain a minimum living standard, measured in terms of caloric intake. In addition to acknowledging the value of these surveys, this article is part of a larger project that seeks to provide theoretical and methodological elements for the understanding of the food issue, and to historically study the development of the science of nutrition.

Key words: food, nutritional surveys, food policies, Chile.

Fecha de recepción: 2 de febrero de 2016. Fecha de aceptación: 17 de mayo de 2016.

* El presente artículo forma parte del Proyecto del Fondo Nacional de Desarrollo Científico y Tecnológico de Investigación Postdoctoral núm. 3160471 titulado Inequidad Alimentaria y Estándar de Vida. Las Políticas de Alimentación Popular en el Chile de entre Guerra: 1920-1950. Agradezco los comentarios de los revisores anónimos que ayudaron a mejorar sustancialmente el artículo.

Am. Lat. Hist. Econ., ene.-abr., 2017, pp. 66-97 | DOI: 10.18232/alhe.v24i1.754 


\section{INTRODUCCIÓN}

$\mathrm{E}$ 1 presente artículo aborda el problema de la alimentación y las dificultades que los trabajadores tuvieron para alcanzar niveles de vida adecuados, medido en términos de consumo de calorías, mediante una serie de encuestas de nutrición que se realizaron entre 1928 y 1938. En concreto, queremos sistematizar los resultados de estas encuestas para determinar patrones de consumo en las familias trabajadoras y así establecer un punto de comparación con el índice del costo de vida (ICV) para la ciudad de Santiago, elaborado por la Dirección General de Estadística (1930) en 1928.

La relación entre el consumo de alimentos y los niveles de vida se originó hace mucho tiempo. El bienestar del que hipotéticamente podían disfrutar amplias franjas de la población en las sociedades tradicionales se medía culturalmente por el acceso que tuvieran a los alimentos considerados fundamentales, como los cereales. Así lo comprueban las revueltas campesinas que se sucedían de manera periódica frente a las crisis de subsistencia del ancien régime (Thompson, 1979).

El desarrollo de la ciencia de la nutrición a fines del siglo XIX transformó el consumo de los alimentos en una variable fundamental para medir el nivel de salud de la población y la noción de "alimentos protectores" para evitar las enfermedades (Marchand, 2014). El concepto de "transición nutricional", por ejemplo, busca explicar la transformación que ocurre históricamente en las sociedades, desde una elevada prevalencia de la desnutrición y patrones de consumo alimentario centrados en los cereales (dieta poco balanceada) a una situación de malnutrición producto de trastornos (obesidad) y patologías crónicas (cardiacas) propias de países desarrollados (Popkin, 1993; Popkin y Doak, 1998; Smil, 2000). En no pocos casos este modelo evolutivo de estudiar la modificación de los patrones alimentarios se acompaña de una transición demográfica y epidemiológica caracterizada por el creciente envejecimiento de la población y la superación de la prevalencia de enfermedades infectocontagiosas, en un marco de aumento del gasto social y consenso en materia de políticas públicas (Cueto, 2000; Ministerio de Salud, 2010; Vargas, 2002).

En términos metodológicos centrarse en el consumo de alimentos tiene efectos comprensivos para entender la evolución de variables demográficas fundamentales, como la mortalidad infantil y la esperanza de vida (Steckel y Rose, 2002), siempre y cuando se incluyan estudios sobre la higiene y la inversión en salud pública. Además, la dieta se considera un factor central en el desarrollo de la estatura y la masa corporal, al estar en relación directa con los ingresos (Cuff, 2004; Fogel, 2009; López-Alonso, 2015). La historia económica ha avanzado lo suficiente frente a la necesidad de sistematizar 
la información recopilada y de proporcionar un conjunto de variables de producción, comercialización y consumo que den cuenta del acceso real a los alimentos, más allá de apreciaciones generales (Fogel, 1993). ${ }^{1}$ Encuestas de consumo familiar, estadísticas de producción global, registros de compras de hospitales, cuarteles o cárceles, menús de escuelas y restaurantes populares, descripciones y testimonios, entre otros, todo ayuda a ese acercamiento (Matus, 2012; Bayardo, 2013). Sin embargo, por su falta de continuidad y limitada representatividad, estas fuentes de información se utilizan de manera cuidadosa para ilustrar determinados análisis.

La medición de la ingesta de calorías para determinar los requerimientos energéticos y nutricionales es heredera de la tradición médica decimonónica preocupada por aumentar el consumo de alimentos y así combatir el desgaste físico en una sociedad en pleno proceso de industrialización (Carpenter, 1994; Hargrove, 2006; Vargas, 2011). Por ello, las estimaciones energéticas de comienzos del siglo XX son variadas, y hay propuestas de ingestas desde las 3000 calorías diarias hasta las 5000 , que varían según el trabajo realizado y dependiendo de si se trataba de un hombre o una mujer (Organización Internacional del Trabajo [OIT], 1936). ${ }^{2}$ En todo caso no faltaron médicos para quienes el equilibrio energético que obsesionaba a algunos colegas ocultaba los problemas asociados a los desequilibrios dietéticos, como una alimentación basada fundamentalmente en cereales.

En este artículo, que forma parte de una investigación mayor sobre alimentación y nutrición en Chile, se constata la falta de investigaciones sistemáticas sobre la evolución del consumo en general y de la alimentación y nutrición en particular. Para la historiadora Inés Pérez (2015), esto se explicaría por la inexistencia de un campo específico de estudio sobre el consumo, lo que ha contribuido al desarrollo de varias disciplinas, pero sin que se centren específicamente en él (p. 99). ${ }^{3}$ Las preocupaciones sobre la alimentación popular se remontan a fines del siglo XIX, con particular énfasis en la producción y distribución de la leche para las mujeres embarazadas, los lactantes y los niños (Zárate, 2013). Al respecto, fueron importantes las gotas de leche y los dispensarios creados por iniciativa del médico Luis Calvo Mackenna, encargados de la atención y crianza

1 Tal como señala Federico (2005, p. 110), si la desnutrición afecta a un número reducido de la población en comparación con décadas pasadas, esto no se debe a problemas de productividad del agro, sino a la distribución de los recursos.

${ }^{2}$ En la actualidad se considera como requerimiento energético una ingesta calórica general de 2500 calorías para los hombres y 2200 para las mujeres.

${ }^{3}$ En cuanto a las investigaciones sobre el consumo en América Latina podemos citar al menos cuatro estudios históricos, Lluch (2015), Milanesio (2014), Rinke (2014) y Torre y Pastoriza (2002). 
de la infancia desvalida (Illanes, 2006). Sin embargo, la cesantía y el aumento del costo de la vida, por efecto de la Gran Depresión, colocaron en el tapete público la necesidad de mejorar la condición del pueblo en su conjunto y no sólo de un grupo. Además de abordar las conocidas "ollas de pobres", se debatió sobre la educación alimentaria, y el Ministerio del Trabajo difundió cartillas con la finalidad de orientar a las personas en cuanto a tipos de alimentos, su valor nutritivo y los costos (Salas, 1932; Yáñez, 2008).

La importancia que comenzó a tener la alimentación como objeto de política pública se explica en gran parte por la relación que se estableció entre ella y el bienestar social, puesto que los alimentos desempeñan un papel fundamental en la salud, el rendimiento productivo y el mejoramiento físico de las personas (Saiki, 1928). En una publicación de 1928, el médico Eduardo Cruz-Coke valoró la alimentación como el principal factor de higiene y salud pública, y utilizó el concepto de "equilibrio alimenticio" para sustentar un enfoque moderno en el análisis de los problemas nutricionales del país. A su vez Robert Debré, miembro del Comité de Higiene de la Sociedad de Naciones, de visita en Chile en 1930, estimó que el principal problema que debía preocupar a las autoridades era el de la alimentación, considerado en sus múltiples dimensiones: higiénica, económica, agrícola y educativa (Debré, 1930).

Las primeras fuentes disponibles en el siglo XX para estudiar las condiciones sociales de la población, y en específico sus niveles de consumo, provienen de las monografías obreras elaboradas por los funcionarios de la Oficina del Trabajo, organismo responsable de levantar la estadística laboral desde 1907. Siguiendo el método de Frederick La Play, las monografías tenían como objetivo estudiar la evolución de ingresos y gastos de una familia tipo, así como la participación que cada uno de los ítems (alimentación, transporte, vestuarios, vivienda, entre otros) tenía en el presupuesto (Oficina del Trabajo, 1911). De esta forma, aunque dichas monografías ayudan a determinar el lugar que ocupaban los alimentos en el gasto familiar, son menos valiosas para calcular su valor nutricional y energético. Parte del problema radicaba en la dificultad de establecer una estructura de consumo representativa de una familia estándar de la época, para lo cual se debían considerar los artículos característicos que se consumían, sus cantidades específicas y las ponderaciones que cada artículo y grupo tenían dentro de la estructura de consumo (Matus, 2012, pp. 57-58).

El artículo se organiza en tres secciones. En la primera se discuten los aspectos metodológicos presentes en la elaboración del ICV de 1928, así como su grado de representatividad para determinar la importancia del ítem de los alimentos en la población capitalina, en especial la más pobre. Si bien fue importante porque proporcionó una canasta actualizada 
y permitió al Estado construir un ICV estandarizado, consideramos que minimizó la importancia que tenían los alimentos en el consumo de los trabajadores, a contracorriente de lo que la literatura contemporánea y los eventos políticos le asignaban. ${ }^{4}$ En la segunda sección se abordan las encuestas de nutrición realizadas entre 1928 y 1938, que forman parte del repertorio cognoscitivo de una segunda generación de médicos preocupados por el estado nutricional de la población (Zárate, 2012). Sugerimos que estos estudios fueron fundamentales para hacer visible la importancia que ocupaban los alimentos en la estructura de gastos de las familias más pobres y los serios inconvenientes que tenían para cumplir con un nivel mínimo de calorías diarias. En un tercer momento se analiza la encuesta que los médicos del Comité de Higiene de la Sociedad de Naciones (SDN) llevaron a cabo en 1935 a solicitud del gobierno de Chile. Además de dar cuenta de las perspectivas transnacionales presentes en organizaciones como la SDN, esta encuesta puede considerarse la más importante del periodo, tanto por sus alcances nacionales como por los aspectos que buscó investigar y que no se limitaron al consumo de alimentos. Por último, se ofrecen consideraciones finales para examinar la construcción de una política de alimentación durante los años de la década de 1930.

Nos resulta fundamental aclarar la noción de familias pobres que muchas encuestas utilizaron y que reproducimos de modo descriptivo. Chile mostró, para el periodo estudiado, amplios niveles de pobreza y desigualdad, con ingresos bajos que afectaban especialmente a los trabajadores no calificados, rurales e informales; por el contrario, el décimo decil concentraba cerca de 55\% de los ingresos totales del país (Rodríguez, 2014, pp. 141249). Si bien el propósito de este artículo no es establecer índices de pobreza para el periodo, las distintas encuestas y estudios realizados entre 1928 y 1938 muestran que las familias pobres dentro del grupo de trabajadores correspondían a aquellas cuyos ingresos no les permitían cubrir sus necesidades básicas, en especial de alimentación, o que debían sacrificar este rubro para pagar otros, como vivienda. En términos sociales eran familias conformadas por un número importante de hijos y allegados, los que no aportaban mayores ingresos al hogar. Por ello optamos por una perspectiva más dinámica y cualitativa de la pobreza, con la cual las encuestas de nutrición nos muestran las condiciones cambiantes que experimentaban las familias, muchas de las cuales en coyunturas económicas favorables

\footnotetext{
${ }^{4}$ Los problemas de producción, distribución y acceso a los alimentos generaron una abundante literatura en paralelo al desarrollo de la "cuestión social" y fueron la causa de numerosos movimientos sociales, como el de 1919 que dio origen a la Asamblea Obrera de Alimentación Nacional, organización de origen multigremial que buscó abordar desde la ciudadanía el problema de las subsistencias en plena crisis salitrera, principal producto de exportación en Chile (Diego, Peña y Peralta, 2002).
} 
pudieron cumplir con una ingesta calórica considerada adecuada e incluso destinar parte de sus ingresos a actividades recreativas. ${ }^{5}$

Las fuentes utilizadas para la elaboración de este artículo corresponden a la revista Estadística Chilena de la Dirección General de Estadística (en adelante DGE), que comenzó a publicar su ICV desde 1928. Las revistas médicas presentaron regularmente investigaciones y debates sobre los problemas de alimentación y muchas de las encuestas nutricionales fueron publicadas por estas revistas o como folletos independientes. Para analizar la encuesta de alimentación que llevó a cabo la SDN en 1935 se usó la versión original en francés publicada en Chile.

\section{EL LUGAR DE LA ALIMENTACIÓN EN EL CONSUMO}

El establecimiento de un ICV se considera fundamental en el diseño de políticas económicas durante la primera mitad del siglo XX en Chile, pues proporciona un indicador relativamente confiable sobre los patrones de consumo y la evolución de los precios y sus efectos en la economía familiar (Díaz, Lüders y Wagner, 1998), además de que se utilizó en los programas de reajuste salarial que se hicieron permanentes a partir de la década de 1930 (Rosemblatt, 1995 y 2000).

Desde fines del siglo XIX la DGE elaboraba un índice de precios al por mayor, para lo cual se seleccionaban algunas ciudades del país y se registraban los valores de productos nacionales e importados y agrícolas e industriales. En febrero de 1928 la DGE comenzó a publicar en su boletín los precios al por menor de la ciudad de Santiago con base en encuestas realizadas en los principales almacenes de la capital, de la Vega y Mercado Central, que clasificaban los artículos en cinco grupos: alimentos, estimulantes, vestuario, combustible y luz, y, por último, varios (DGE, 1928a, p. 46). Llama la atención la diversidad de artículos que incluía el grupo de alimentos, los que alcanzaban el número de 34, si se suman los dos primeros subgrupos. Es importante hacer notar que dentro de los estimulantes se encontraban el café, el té, la yerba mate, la cerveza, el vino y los cigarrillos. En la lista, además, dentro del grupo varios, estaban artículos de aseo (jabón), cultura e información (compra de periódicos) y actividades recreativas (entradas al cine).

Diversos estudios han determinado las condiciones complejas que enfrentaron los trabajadores chilenos durante la Gran Depresión y se estima que los salarios reales recuperaron su valor anterior a las crisis de 1929 apenas en 1945, aunque se mostraron estables y hacia un alza creciente a partir de 1938. Véanse, por ejemplo, Reyes (2015) y Rodríguez (2013). 
Sin embargo, en noviembre de 1928 la DGE dejó de publicar los precios al por menor, y los reemplazó por el ICV para la ciudad de Santiago. Si bien desde 1923 hasta fines de 1927 la DGE había entregado un ICV, los precios que servían de base para establecer ese cálculo incluían artículos al por mayor y al menudeo, además de dejar muchos productos fuera del registro e incluir otros no del todo representativos del consumo de las familias. Considerando estos antecedentes, la DGE decidió elaborar un libro sobre los gastos del hogar, el cual debía ser repartido entre varias familias para establecer de manera más exacta la importancia relativa de cada uno de los artículos. Mientras se llevaba a cabo esa encuesta, se tomó "como base a una familia de tres personas adultas o de dos adultos y dos menores de diez años, cuya renta es de $\$ 600$ mensuales" (DGE, 1928b, p. 354). Siguiendo la clasificación de los precios al por menor, los artículos de consumo se dividieron en cinco grupos, aunque para determinar su ponderación se recurrió a la opinión de expertos ("personalidades experimentadas en la materia") y se obtuvieron los siguientes valores: la alimentación correspondía a 45\% del gasto total de un hogar; la habitación, a 20\%; los combustibles y luz, a 7.5\%; el vestuario, a 15\%, y el grupo varios, a $12.5 \%$. Para determinar la ponderación del grupo de alimentos, la DGE optó por encuestar a las propias familias de sus empleados (un total de 68 personas adultas y seis menores de diez años), y se determinó un promedio de consumo de cada artículo (véase cuadro 1).

Si nos concentramos en el cuadro 1, la canasta de 1928 estableció en el grupo de alimentos un número de 26 artículos, un poco más reducido que el índice de precios al por menor que comprendía 34 productos. La diferencia radica en que este último índice incluía aceite de soya, distintos tipos de pescado y carne, y duraznos al jugo. Además, diferenciaba la leche fresca de la condensada, y comprendía diversas verduras y hortalizas que en el ICV de 1928 estaban incorporadas en las legumbres. La importancia de estas últimas no es menor, considerando que tenían la segunda ponderación, luego de la carne, con 13.6 por ciento.

No tenemos claro por qué la DGE integró las legumbres con las verduras, sobre todo si reconoció la importancia de elaborar cada mes un índice especial de verduras y hortalizas, que incluía ají verde, apio, arvejas, ajos, alcachofas, cebollas, choclos, lechugas, porotos verdes, repollos, tomates, zanahorias, zapallos y coliflores (DGE, 1929). Esto no sólo demostraba lo regular que era el consumo de estos productos por parte de las familias de la capital, aunque disminuyera su ingesta durante algunas semanas o meses, sino también lo sensibles que podían ser los hogares a sus cambios de precios (véase cuadro 2). La evolución del índice general del subgrupo verduras y hortalizas para 1928 da cuenta de alzas importantes a partir de julio hasta noviembre, en especial en los tomates, cebollas y zapallos. 
CUADRO 1. GRUPO ALIMENTOS. CANASTA DE 1928

\begin{tabular}{lclc}
\hline Alimentos & Porcentaje & Alimentos & Porcentaje \\
Harina & 0.6 & Queso & 0.8 \\
Pan & 8.9 & Manteca & 4.1 \\
Arroz & 1.7 & Huevos & 5.5 \\
Frijoles & 0.7 & Carne & 22.6 \\
Papas & 2.6 & Carne & 3.9 \\
Fideos & 0.5 & Pescado & 1.6 \\
Mote & 0.2 & Huesillos & 0.5 \\
Azúcar & 2.7 & Té & 5.5 \\
Sal & 0.3 & Café & 2 \\
Aceite & 3.2 & Yerba mate & 4 \\
Leche & 7.7 & Vino & 1.6 \\
Mantequilla & 1.9 & Cerveza & 0.3 \\
Cigarrillos & 3 & Legumbres & 13.6 \\
\hline
\end{tabular}

Fuente: DGE (1930).

No deja de sorprender la importancia asignada a la carne dentro del ICV de 1928, que representa $26.5 \%$ del total de los gastos en alimentos, mientras que al pan se le asignó una ponderación de sólo 8.9\%. Esto indica que las familias utilizadas como referencia eran mayoritariamente empleados que destinaban un mayor presupuesto al consumo de carne, a diferencia de las clases trabajadoras que aumentaban el consumo de pan o derivados del trigo, teniendo en cuenta su mayor eficiencia en la relación costo-calorías. ${ }^{6}$ Lo anterior significa que, al tomar como referencia a una familia tipo de Santiago, con un ingreso mensual de 600 pesos, y basarse en el consumo característico de algunos hogares de empleados para determinar el número y la ponderación de los artículos del grupo de alimentos, se generaron algunas distorsiones que terminaron por afectar el grado de representatividad del ICV. El mismo Instituto Nacional de Estadística (1957), en su índice de precios al consumidor (IPC) de 1957, reconoció lo bajo de la ponderación atribuida a los alimentos en 1928, y le asignó en el nuevo indicador un porcentaje de $47 \%$, es decir dos puntos más que en 1928: "Por otra parte, como se ha dicho anteriormente, el Índice de 1928 fue construido a base de estudios de familias de empleados. Pero los pro-

${ }^{6}$ Cien gramos de pan aportan 280 calorías aproximadamente, mientras que 100 gramos de pollo, 120 calorías y 100 gramos de carne de vacuno, 190 calorías. 


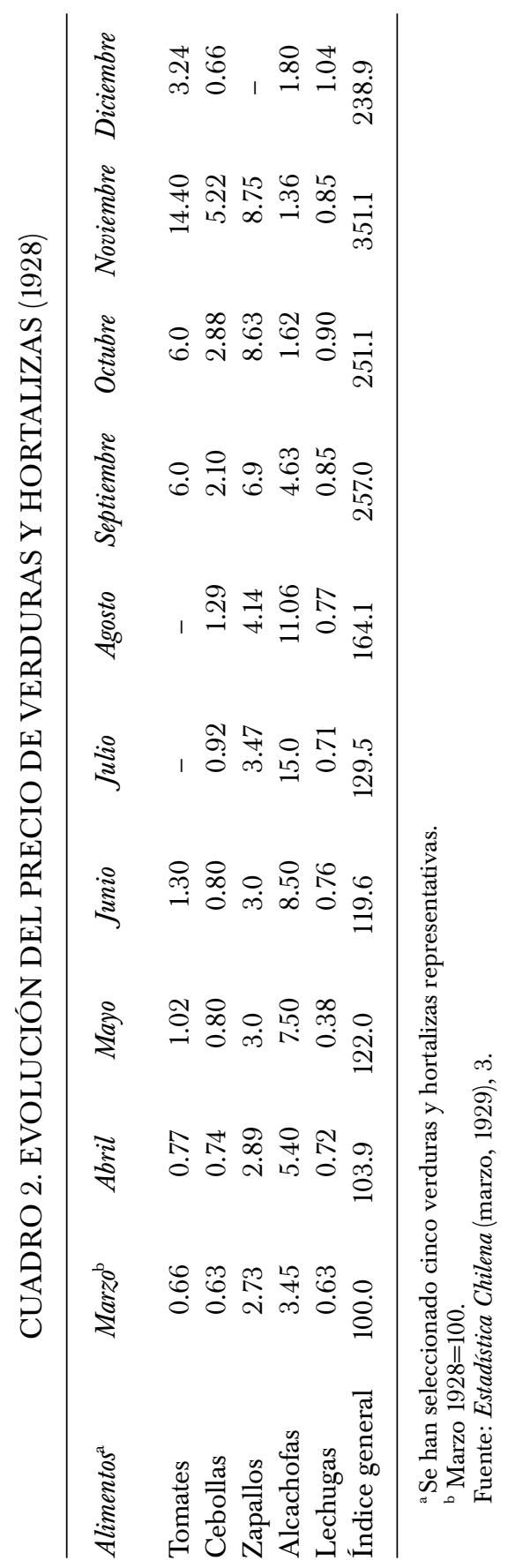


blemas económicos y muchos de los sociales de la clase obrera, a través del tiempo fueron solucionándose, en su mayor parte teniendo también como indicador del movimiento del costo de la vida este mismo Índice. Este hecho justificaba, por sí solo, que el Índice [el de 1957] cubriera ambos estratos sociales" (p. 2).

En nuestro caso, lo que más nos interesa destacar es la subrepresentación que tenía el grupo de alimentos en la canasta de 1928 al asignarle sólo $45 \%$ de los gastos totales. Esto contradice las encuestas de nutrición del periodo que habían situado el gasto en alimentación de las familias trabajadoras en al menos 60\% (véase gráfica 1), lo que refleja los rasgos tradicionales que tenía la sociedad santiaguina del periodo y su orientación hacia el consumo primario de productos, tal como lo indica la ley de Engel.

Aunque en la época era muy difícil conformar una canasta representativa del conjunto de la población, si consideramos que el grupo de los alimentos fue el que más sufrió variaciones durante el primer año de aplicación del ICV (véase cuadro 3), al disminuir la participación de este grupo en el gasto total de las familias se hizo menos visible el impacto que tenían los altos precios de los alimentos en los sectores más pobres. Tal como la literatura señala al respecto, para la determinación de un IPC validado se debería cumplir al menos con tres requisitos: $a$ ) los datos utilizados deben corresponder a precios finales, es decir pagados por los consumidores; b) los datos deben incluir un número variado y representativo de bienes y servicios; $c$ ) que estos bienes y servicios hubiesen sido seleccionados $\mathrm{y}$ ponderados de acuerdo con las pautas reales del gasto de las familias $\left(\mathrm{Ca}^{-}\right.$ rreras, Hofman, Tafunell y Yáñez, 2003). En especial este último punto no se cumplía del todo en el ICV de $1928{ }^{7}$

Si se hace una corrección en el ICV de 1928 con base en una representación más real del grupo de alimentos en la estructura de gastos de las familias trabajadoras, de acuerdo con la información proveída por siete encuestas de nutrición entre 1928 y 1937 y los estudios sobre consumo de la Oficina del Trabajo de 1911, los alimentos comprenden en promedio $70.5 \%$ del gasto total del consumo hogareño, muy cercano al $70 \%$ que le asignó la encuesta de alimentación del Comité de Higiene de la SDN de 1935, considerada una de las más completas del periodo. De esta forma hemos tomado como referencia dicha investigación para determinar la estructura de gastos de las familias trabajadoras del periodo, y hemos asignado los siguientes valores: $70 \%$ a la alimentación; $10 \%$ a la habitación; $10 \%$ a los combustibles y la luz; $5 \%$ al vestuario, y $5 \%$ a varios. Con estos

\footnotetext{
${ }^{7}$ Las cifras oficiales de la DGE muestran que la inflación tuvo una variación anual promedio, entre 1928 y 1938 , cercana a $6 \%$, con una fuerte alza en $1932(23.6 \%), 1936(12.3 \%)$ y $1937(9.8 \%)$.
} 


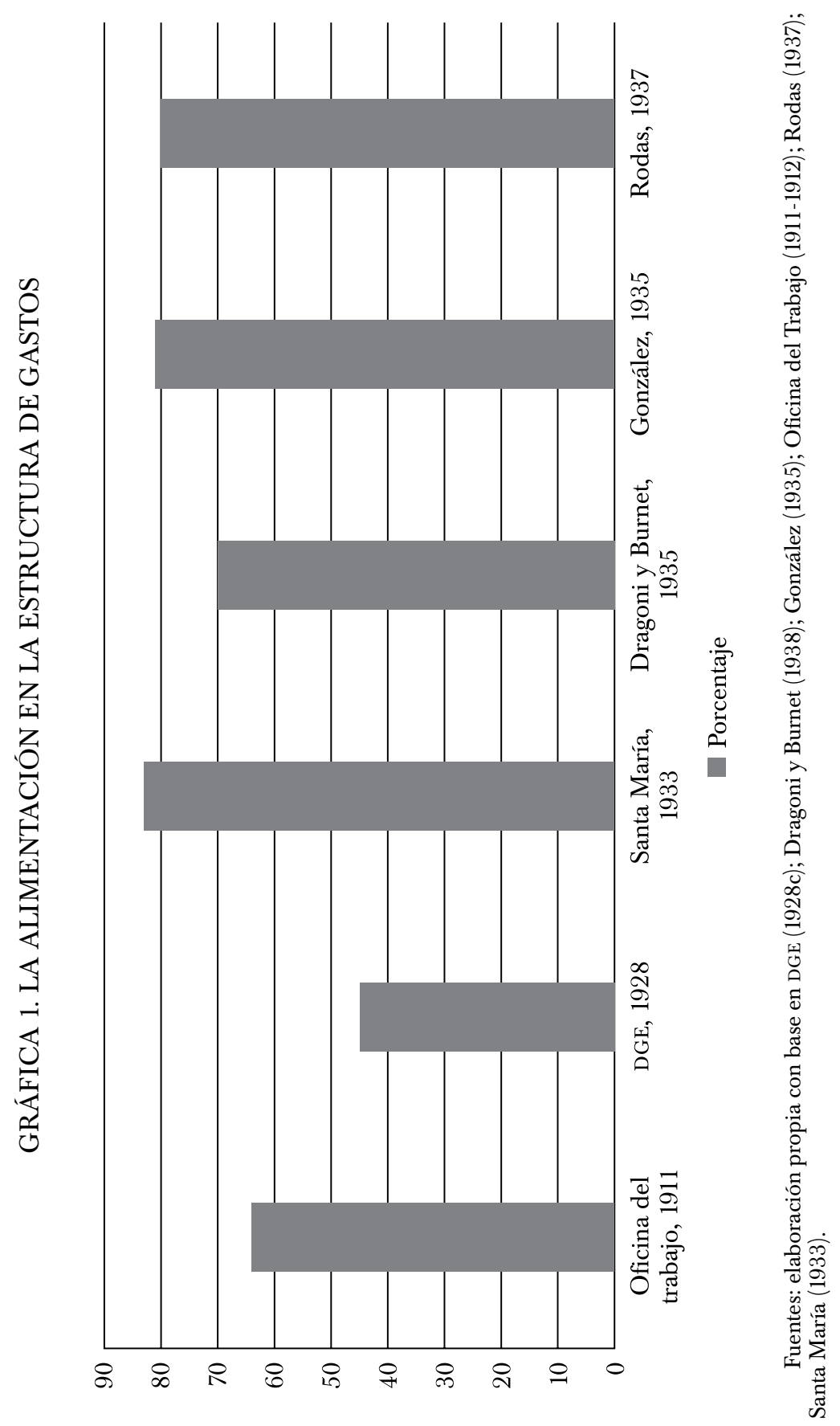




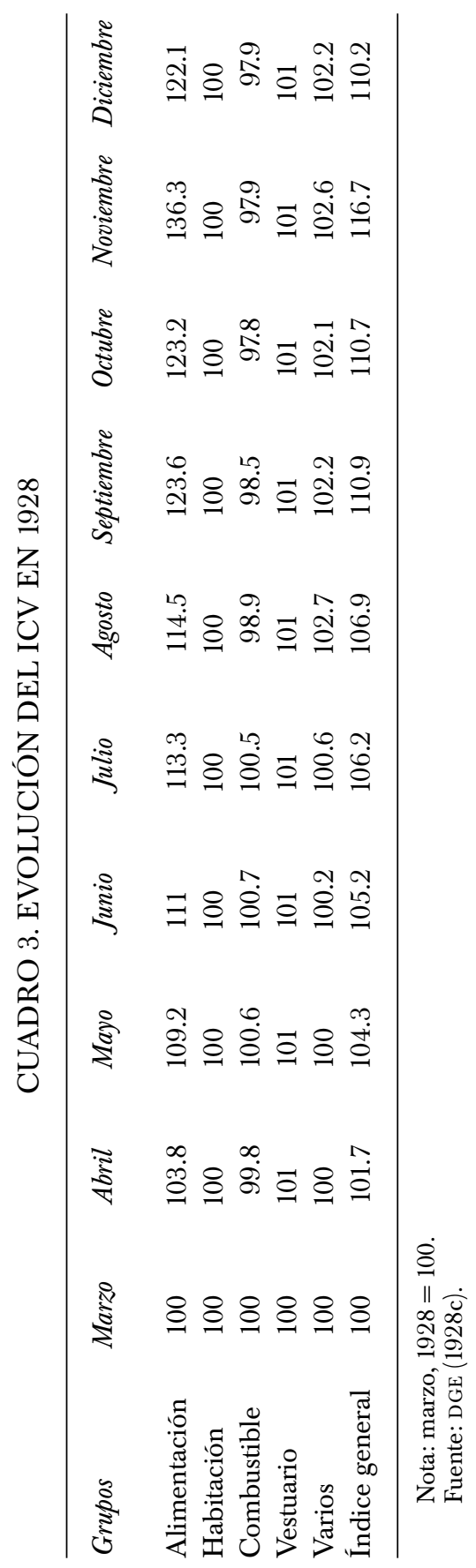


datos se puede corregir el ICV de 1928, reinstalando la alimentación como parte importante del consumo familiar durante los años 1930, de lo que resultan las siguientes cifras de la gráfica 2 , que marcan una distancia entre el ICV de la DGE y el ICV corregido por nosotros.

Tal como indica esta gráfica, las diferencias entre el ICV de la DGE y el ICV corregido son consistentes con las variaciones experimentadas en el grupo de alimentos en los meses de mayo, septiembre, octubre y noviembre de 1928, con diferencias de hasta nueve puntos en noviembre.

En síntesis, la canasta que la DGE estableció en 1928 para determinar el ICV redujo la importancia de la alimentación en los gastos de las familias; por el contrario, las encuestas de nutrición fueron las que mostraron de manera más fidedigna la relevancia que tenían los alimentos en el consumo, especialmente de los más pobres. Esto llevó a la comunidad médica y a las autoridades a acordar la formulación de políticas alimentarias y con ello no sólo enfrentar los problemas nutricionales, sino también generar una transformación cultural del país.

\section{LAS ENCUESTAS DE NUTRICIÓN}

Un giro que marcó las investigaciones sobre la alimentación fue la aplicación de las primeras encuestas de nutrición hacia fines de los años veinte, lo que permitió estandarizar la información recopilada y compararla en distintos periodos. En la época existían dos modalidades para determinar la ingesta de calorías. En primer lugar, el uso de las cuentas nacionales, en que se calculaba la producción alimentaria del país, por lo general durante un año, restando las exportaciones y sumando las importaciones, para luego dividirla entre la cantidad de habitantes. La cifra final en gramos se transformaba en calorías de acuerdo con coeficientes de composición nutricional de los alimentos. La ventaja de esa metodología era que permitía, mediante el balance de alimentos, acceder a información previamente clasificada y validada, y obtener un resultado de oferta de calorías independiente de los factores locales. Su desventaja radicaba, precisamente, en que las cifras resultantes eran demasiado abstractas como para orientar una política focalizada de alimentación.

Los médicos chilenos optaron la mayoría de las veces por el método de la encuesta, la cual se aplicaba a un número de familias representativas de un segmento de la sociedad (clase alta, clase media y clase trabajadora), registrando el consumo diario y estableciendo la unidad hombre día (UHD), es decir la ingesta de alimentos en adultos y menores según una ponderación dada por la edad de sus miembros. Sin embargo, por su falta de continuidad y limitada representatividad, estas encuestas deben usarse 


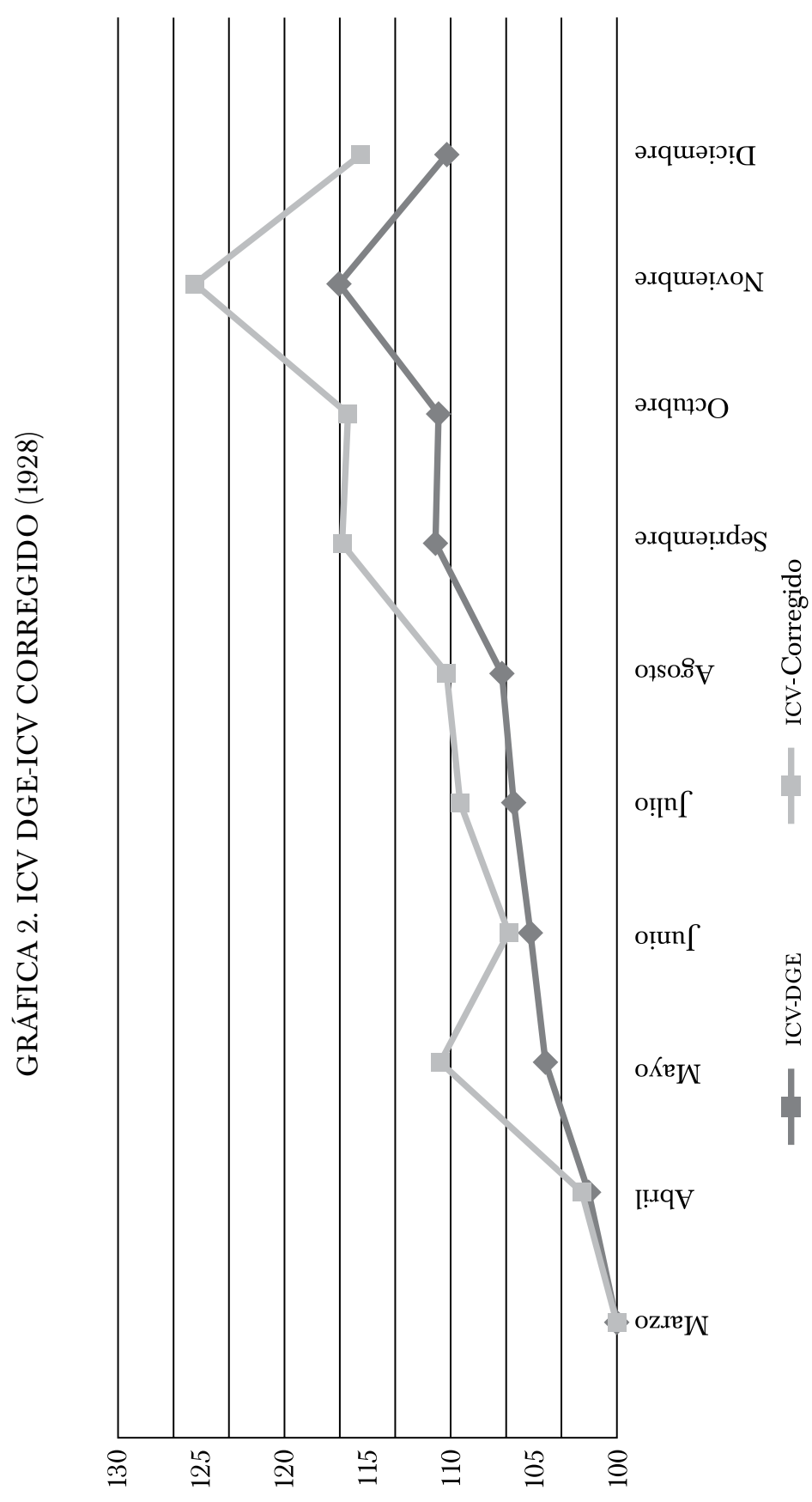

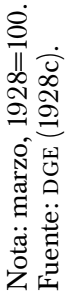


como fuentes de información general que ayudan a ilustrar determinados análisis, sin extrapolar sus resultados. Otro aspecto que mueve a engaño es la estimación de las necesidades energéticas que se consideraban adecuadas para responder a las exigencias de la vida moderna. Por ejemplo, la medicina de guerra, interesada en preparar cuerpos de tropas resistentes, propuso un consumo diario cercano a las 5000 calorías, difícil de alcanzar por el resto de la sociedad. En 1935, el Comité de Higiene de la SDN, reunido en Londres, determinó una ingesta de al menos 2400 calorías para la mantención de la salud de una persona que vivía en un clima templado y ejercía alguna actividad moderada, a lo cual se debían agregar 50 calorías diarias por cada hora de trabajo mediano o bien 100 calorías en aquellos intensos. En 1936, la Organización Internacional del Trabajo (OIT), en una conferencia regional llevada a cabo en la ciudad de Santiago, fijó la ingesta de calorías mínimas en 3000 diarias y en 1950 la Organización de las Naciones Unidas para la Agricultura y la Alimentación (FAO) propuso valores de referencia de 3200 para el hombre y 2300 para la mujer (OIT, 1936; Bourges, Bengoa y O'Donnell, 2002). Por último, las tablas calóricas aplicadas correspondían a las utilizadas en Europa y difundidas por el Comité de Higiene de la SDN, las que eran replicadas en revistas de medicina (Tablas de alimentación, 1938, pp. 30-31). En muchos casos las encuestas no aportan mayor información sobre el tipo y calidad de los productos consumidos, entre los cuales la carne, por ejemplo, se presta más a confusiones, porque no está claro si se trataba de carne de vacuno, de cerdo o de pollo.

De todas formas, y más allá de las limitantes metodológicas, los médicos estaban conscientes de que los regímenes calóricos no eran suficientes para medir una dieta balanceada, si esta no incluía proteínas, grasas e hidratos de carbono. El médico Julio Santa María (1935) señaló al respecto:

En algunas circunstancias, pues, forzando la ingestión de algunos alimentos que producen muchas calorías se puede llegar a un falso equilibrio nutritivo que parece compensar el trabajo muscular, cuando en realidad no repara el desgaste corporal del individuo ni atiende al buen funcionamiento de su salud, de su actividad intelectual, ni de su actividad genésica. Para todas estas funciones se necesita imprescindiblemente un mínimum de proteínas (p. 5).

Como muchas encuestas buscaban establecer la relación entre el régimen alimenticio y la condición socioeconómica, por lo que se centraban en las poblaciones de escasos recursos y trabajadores; su interés radica en poder determinar ciertos patrones entre el aporte calórico de los alimentos y su relevancia en el presupuesto total. De este modo se llegó a la conclusión de que muchas familias daban prioridad a productos altamente calóricos pero que no tenían un alto impacto en su presupuesto. 
Por otra parte, las encuestas tendieron a tomar como unidad de análisis a la familia y no al individuo aislado. En un enfoque netamente sociológico, que marcaría el desarrollo de la nutrición y de las políticas de alimentación en Chile, el hogar se consideraba el núcleo central en la formación de hábitos alimenticios y el origen de la mayoría de los trastornos nutricionales (Lipschutz, 1933; Santa María, 1949). Algunos médicos vieron en la desestructuración de la familia en el contexto de la llamada cuestión social la causa de numerosas patologías y en especial de la mortalidad infantil (Dagnino, 1928).

Una variable relevante era el número de miembros que componían el hogar, pues en no pocos casos se encontró una relación directa entre el aumento en la cantidad de niños y las deficiencias alimenticias. Eduardo Cruz-Coke (1928) hizo hincapié en la relación existente entre el desequilibrio dietético y el incremento de enfermedades y patologías, como el raquitismo y la tuberculosis, las cuales afectaban especialmente a niños, debido a que muchos padres al enfrentarse a una situación de crisis reducían la ingesta de alimentos de los menores.

La primera encuesta de nutrición en Chile parece ser la de Eduardo Cruz-Coke, realizada en 1928 en el marco de su curso de nutrición, y publicada en la Revista Médica de Chile. Lo interesante es que en ella se comparan los resultados de una encuesta aplicada a 50 familias de diversas clases sociales con aquellos obtenidos analizando las cuentas nacionales o la oferta alimentaria disponible, de modo que se obtuvo un consumo de 2600 calorías diarias para la primera y de 2918 para la segunda. La comparación de ambos métodos daba como resultado una cifra intermedia para el país de 2750 calorías por persona, aunque limitada, a decir del mismo Cruz-Coke, porque partía de datos estadísticos optimistas y no tomaba en cuenta el problema de la distribución de los alimentos.

Los estudios nutricionales basados en las cuentas nacionales fueron seguidos por Raquel Reyes (1933) quien en el marco de una memoria de prueba analizó las estadísticas de producción y consumo agrícola y ganadero entre 1912 y 1931. De acuerdo con estos datos el valor energético del régimen alimenticio medio había aumentado desde las 1760 calorías por día en 1912 hasta 2350 en 1931, en plena crisis económica, y alcanzó su punto más alto en 1928 con 2650 calorías. Pese a los cuestionamientos que se hicieron a su metodología -véase Mardones (1935, p. 369)-, en especial el utilizar cifras de producción y consumo no del todo fiables, lo que mostraba la investigación de Raquel Reyes era la baja oferta calórica de que disponían las familias chilenas (no sólo trabajadoras) a comienzos de la década de 1910, hasta alcanzar un consumo considerado aceptable a fines de los años veinte, antes de la crisis (véase gráfica 3). 


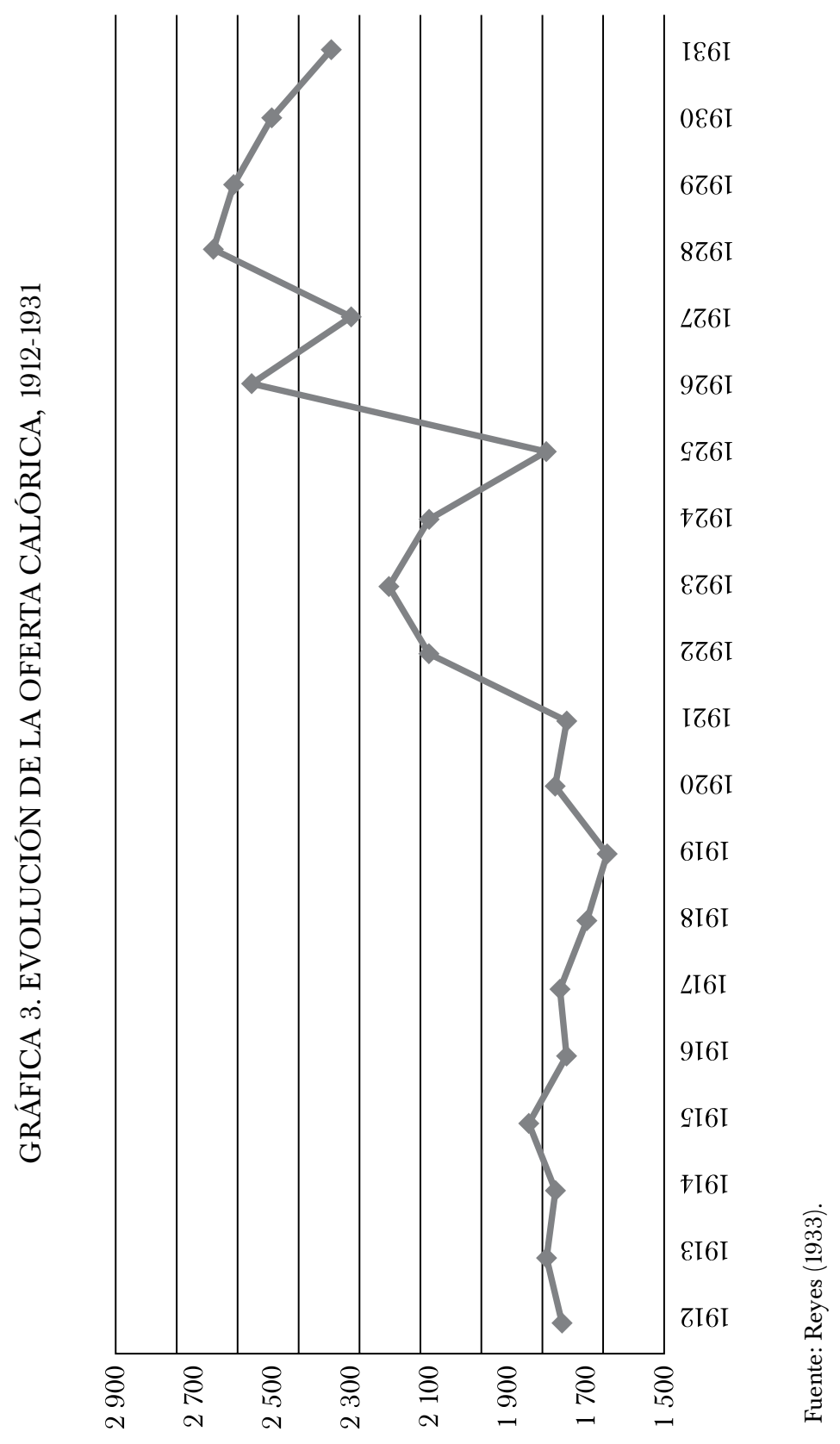


Si se analizan con más detalle los resultados de Raquel Reyes, podemos señalar que estas cifras son consistentes con las variaciones presentadas por el PIB per cápita en el mismo periodo, en la medida en que los datos de calorías basados en las cuentas nacionales dependen principalmente de los niveles de producción, en especial de productos agropecuarios. Por ejemplo, las bajas en la oferta de calorías de 1919 y 1927 se pueden explicar por la disminución del PIB per cápita de - $15 \%$ y de $-3.10 \%$ respectivamente, mientras que las caídas de 1925 y 1929 pueden deberse a la disminución de la producción agrícola, la cual no se recuperaría sino hasta 1933 (Braun et al., 2000, pp. 23-25). Todo lo anterior limitó el acceso a determinados alimentos o sacrificó su reparto en las familias. ${ }^{8}$ Esto es más notorio en la disminución de calorías para 1930 y 1931, que coincide con las variaciones del PIB per cápita de $-17.24 \%$ para el primer año y de $-22.36 \%$ para el segundo (Braun et al., 2000, p. 23). Los incrementos en la oferta calórica muestran comportamientos similares en su relación con el PIB per cápita, en especial entre 1921 y 1924, los que se pueden explicar por una recuperación del PIB per cápita de entre 2.31 y 18.88\%. Por último, las alzas en la oferta de calorías de 1926 y 1928 coinciden con un aumento de la productividad agrícola para el primer año y con un alza del PIB per cápita de 20.91\% para el segundo (Braun et al., 2000, pp. 23-26).

El médico Julio Santa María (1933, p. 5) llevó a cabo una encuesta entre 48 familias trabajadoras de la Refinería de Azúcar de Viña del Mar, la que arrojó un consumo promedio de 3275 calorías por día, una cifra mayor a la media de las encuestas anteriores, lo que indica que se analizó la situación de un sector favorecido de la clase obrera. Por ejemplo, las familias tenían un salario promedio de trece pesos diarios y recibían subsidios en habitación, agua, luz y azúcar. Pese a lo anterior, según Santa María, $80 \%$ de las familias no consumía una ración acorde a los requerimientos energéticos de su trabajo, establecido en 3500 calorías, y sólo lo hacían aquellas que tenían un mayor ingreso o menor número de hijos. El consumo de leche era muy reducido, representaba sólo 5.6\% de las calorías totales, y el consumo de carne llegaba en promedio a 187.7 gramos por día, lo que correspondía a 8\% del aporte calórico total. Esta encuesta de 1933 es una de las que asigna un porcentaje menor al consumo de pan, $27.3 \%$ del aporte energético total, lo que se explica por el alto consumo de azúcar gracias a los beneficios que los trabajadores obtenían de la empre-

${ }^{8}$ La gran mayoría de las encuestas y estudios del periodo explican los niveles de desnutrición o subalimentación que presentaban las familias, especialmente los niños, a partir del número de sus integrantes, y concluyeron que en situaciones críticas el padre restringía la ingesta de alimentos a sus hijos menores, quienes, paradojalmente, más necesitaban alimentos, pero no aportaban económicamente al hogar. 
sa, con un consumo por persona de 141 gramos, que aportaban $17 \%$ del total de calorías.

Otro médico, Ramón González (1935), publicó los resultados de una encuesta realizada a 90 familias obreras de Santiago, y diagnosticó una subalimentación crónica en el contexto de la lenta recuperación de la economía luego de la Gran Depresión. Los aportes calóricos alcanzaban en promedio 2540 calorías por día, que de acuerdo con el rango óptimo de 3000 mostraba un déficit cercano a 15\%. El problema se agravaba en los hogares que tenían niños menores de catorce años, donde se reducía el consumo de proteínas animales (carne, huevo y leche) en 50\%. La ingesta de leche era mínima; de las 90 familias sólo 62 la consumían, aunque en cantidad pequeña (127 gramos por UHD), correspondiente a $2.7 \%$ del régimen total energético, comparado con el pan, que alcanzaba $35.9 \%$ (véase cuadro 4).

Esta encuesta, al presentar datos detallados, permite analizar las estrategias que los sectores más pobres utilizaron para hacer más eficiente el uso de sus ingresos. El pan es un buen ejemplo de esto por la excelente relación que tenía entre costo y aporte energético: contribuía con $35.9 \%$ de las calorías diarias, pero representaba sólo 23.8\% del gasto total. Es decir, se consumía mucho pan porque era más barato y eficiente en relación con el aporte energético de otros productos. Por el contrario, el consumo de carne era más bien bajo, pues aportaba sólo 9.7\% del valor energético total, pero representaba $28.4 \%$ del gasto presupuestario diario. En concreto, para consumir 248 calorías de carne se debía gastar cerca de $28.4 \%$ de los ingresos diarios. Por otra parte, no sorprende la importancia de las papas, con un aporte energético de $10.4 \%$, se sitúa como el segundo producto más consumido. El poco consumo de verduras se explica en parte porque tenían un aporte calórico reducido, de 3.1\%, pero representaban un costo de $9.2 \%$ del presupuesto total.

El médico Ángel Rodas (1937) publicó los resultados de una encuesta aplicada entre los sectores más pobres de la ciudad de Chillán, localidad situada a 450 kilómetros al sur de la capital, con un universo total de 150 familias, pero sólo se pudo reunir información completa de 36 de ellas. El promedio de calorías consumidas era de 1832 y sólo dos familias recibían un mínimo de 3000 diarias. El consumo de carne era inexistente, las proteínas animales representaban sólo 1.3\% del aporte calórico total. En tanto los hidratos de carbono alcanzaban la cifra desproporcionada de 79\%, y el trigo (en forma de pan, harina, mote y fideos) cubría $47 \%$ del total de calorías.

Por último, Inés Torres (1938), en el marco de su memoria de prueba, publicó un estudio sobre la alimentación popular según los datos de censos demográficos y agropecuarios, en el cual concluyó que la oferta de calo- 
Am. Lat. Hist. Econ., año 24, núm. 1, enero-abril, 2017, pp. 66-97

\section{CUADRO 4. COMPOSICIÓN Y COSTO DEL RÉGIMEN MEDIO}

\begin{tabular}{lrcc}
\hline \multirow{2}{*}{ Alimento } & \multicolumn{2}{c}{ Valor energético } & Costo \\
& Calorías & Porcentaje régimen total & Porcentaje régimen total \\
Carne & & & \\
Leche & 248 & 9.7 & 28.4 \\
Pan & 70 & 2.7 & 4.2 \\
Papas & 913 & 35.9 & 23.8 \\
Grasa & 264 & 10.4 & 5.8 \\
Mantequilla & 198 & 7.8 & 4.2 \\
Verduras & 9 & 0.4 & 0.4 \\
Vino & 80 & 3.1 & 9.2 \\
Fideos & 38 & 1.4 & 3.3 \\
Harina & 173 & 6.2 & 4.2 \\
Frijoles & 61 & 2.4 & 1.2 \\
Azúcar & 200 & 7.9 & 2.9 \\
Aceite & 164 & 6.4 & 4.2 \\
Arroz & 18 & 0.7 & 0.8 \\
Varios & 94 & 3.7 & 3.7 \\
& 18 & 0.7 & 3.7
\end{tabular}

Fuente: González (1935).

rías disponibles por unidad de consumo para 1935 correspondía a 1923 calorías $^{9}$ (véase cuadro 5). El consumo de trigo y de papas seguía siendo predominante; el primero representaba más de 50\% del total del aporte calórico. Si bien los aportes calóricos del azúcar y de la carne aparecen representados en exceso, con 60 y 40 calorías respectivamente, esto se compensa por el hecho de que la autora no incorporó el consumo de vino en la tabla. Todos los autores indicaban que el consumo de alcohol estaba extendido entre los trabajadores y era un importante componente en la dieta, que podía aportar 70 calorías por 100 centímetros cúbicos.

En síntesis, más allá de las deficiencias halladas en muchas de estas encuestas y del grado de representatividad que tuvieron, ayudaron a fundamentar la formulación de políticas de alimentación a partir de la segunda mitad de la década de 1930. En general mostraron los desequilibrios crónicos en el consumo de las familias trabajadoras y cómo la mayoría

\footnotetext{
${ }^{9}$ Aunque en el texto original aparece un cálculo total de 1996 calorías, producto evidente de un error en la sumatoria (Torres, 1938, p. 49).
} 
CUADRO 5. UNIDAD DE CONSUMO, 1935

\begin{tabular}{lcc}
\hline Alimentos & Cantidad (gramos) & Calorías \\
Trigo y derivados & 324 & 1085 \\
Carne & 52 & 128 \\
Leche (cc) & 75 & 50 \\
Papas & 200 & 134 \\
Azúcar & 60 & 288 \\
Aceite y grasas & 9 & 8 \\
Leche (derivados) & 6 & 30 \\
Garbanzo, lentejas y arvejas & 12 & 39.7 \\
Arroz & 11 & 38.5 \\
Pescado & 15 & 10.4 \\
Porotos & 35 & 112 \\
Total & & 1923 \\
\hline
\end{tabular}

Fuente: Torres (1938).

de estas no alcanzaba un aporte energético considerado suficiente para el periodo (véase gráfica 4 ).

La gráfica 4 muestra que las 2350 calorías por persona estimadas por Reyes (1933) para 1931 (utilizando el sistema de cuentas nacionales) se mantuvieron relativamente estables para la década de 1930, y no recuperaron el nivel de 1928 (2 650 calorías), a excepción de la encuesta de Julio Santa María de 1933 aplicada a un sector privilegiado de los trabajadores (con 3275 calorías). Los bajos índices mostrados en la encuesta de Rodas en 1937 (1 832 calorías) se explican, como se dijo anteriormente, porque se encuestó a familias pobres de la zona rural de Chillán. Respecto al estudio de Torres (1938), sus resultados muestran, utilizando la misma metodología de cuentas nacionales de Reyes, que los niveles de producción y consumo agropecuario no presentaron signos de recuperación luego de la gran depresión que hicieron sostenibles los cambios demográficos y migratorios que experimentaba la sociedad chilena en esos años. ${ }^{10}$

En otro aspecto, las encuestas muestran el excesivo consumo de trigo, que alcanzó un promedio de $42 \%$ de la ingesta calórica total, aunque en algunos casos, como en Cruz-Coke (1928) y Torres (1938), podían lle-

${ }^{10}$ Por ejemplo, entre el censo de 1930 y 1940 la población rural disminuyó de 51 a 47\%; su fuerza de trabajo de 38.9 a $36.9 \%$ y la productividad agrícola (PIB en millones de pesos de 1995) sólo aumentó de 348000000 en 1928 a 358000000 en 1938. Véase Braun et al. (2000, pp. 27-29). 


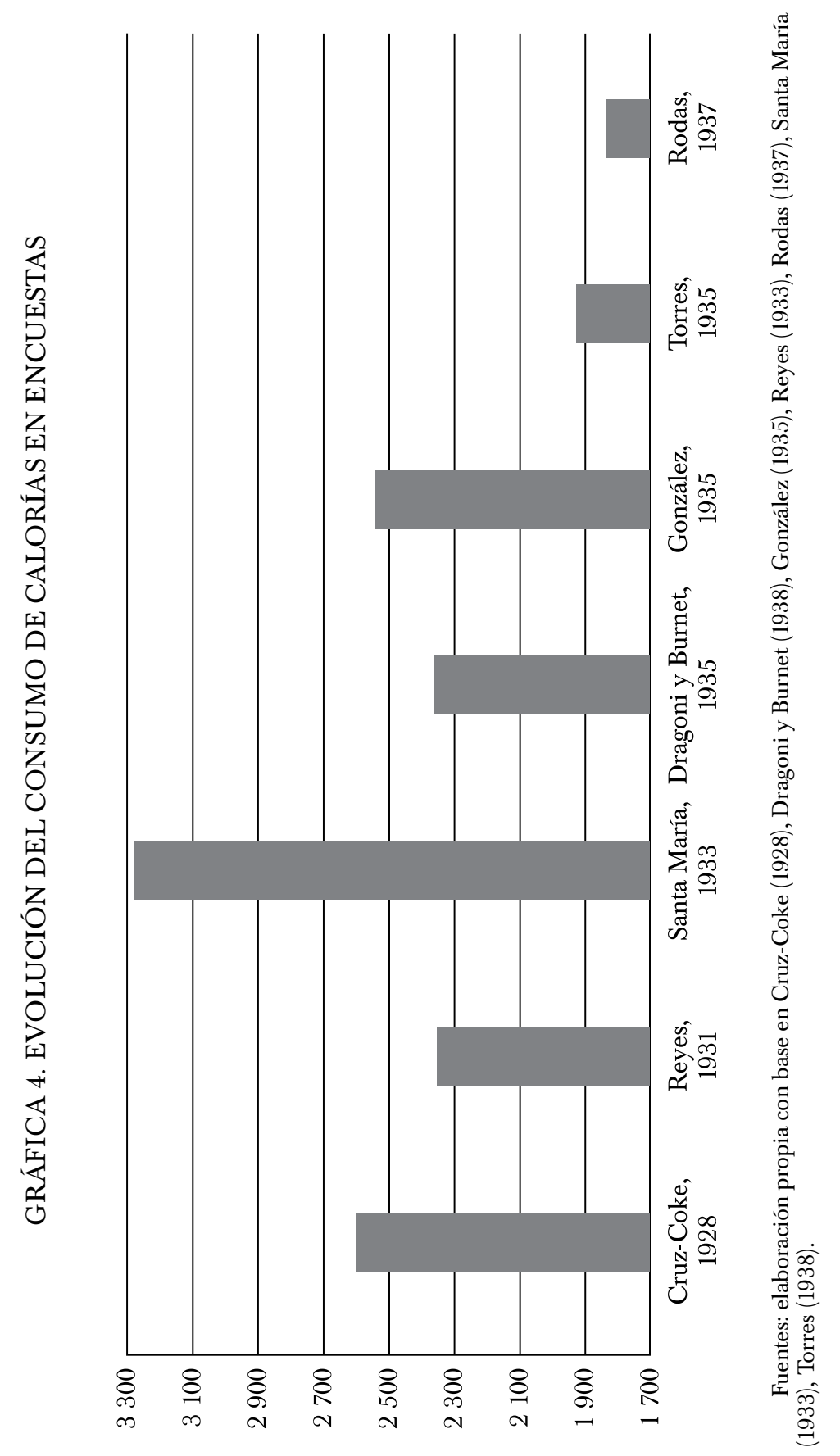


gar a 50\%. Una dieta basada mayoritariamente en los cereales parecía ser consecuencia de las condiciones económicas deficientes y no de aspectos culturales. Si bien el trigo era lo bastante dúctil como para ser consumido de distintas formas (mote, harina tostada y pan), lo más importante es que era barato en relación con otros productos: "A medida que las condiciones económicas de las familias mejoran, los gastos en cereales continúan aumentando, pero a una tasa decreciente, de tal manera que terminan por estabilizarse" (Dragoni y Burnet, 1938, p. 175). El hecho de que haya primado un enfoque sociofamiliar en los estudios sobre el estado nutricional de la población trabajadora (incluso en aquellas encuestas que se realizaron dentro de las industrias) obligó a establecer relaciones precisas entre las deficiencias alimenticias y la composición de los hogares. De esta manera se concluyó - con una mirada maltusiana- que había una relación directa entre el estado nutricional de las familias y el número de menores que la componían, sin dejar de lado problemas de desorganización presupuestaria y desconocimiento de nociones económicas básicas. Quizá sea interesante contrastar con los datos de ingesta de calorías que presenta Julio Santa María (1935, p. 11) para el complejo cuprífero de Sewell, y que eran 4226 por unidad de consumo. Este alto valor, si lo tenemos por cierto, único en la década de 1930, se explica tanto por las políticas promovidas desde la empresa, interesada en cumplir con los requerimientos energéticos de trabajadores expuestos a actividades extractivas (Vergara, 2008; Garrido, 2014), como por la poca cantidad de hijos que tenían las familias, producto de los estrictos códigos morales existentes (Klubock, 1998). Esto último hizo que las tablas de consumo presentaran un bajo promedio de integrantes por familia, como la de 1936 con 3.2 integrantes (Seibert, 1936, p. 51).

Muchos de estos análisis se enriquecieron con las conclusiones a las que llegó una de las encuestas más importantes del periodo, la aplicada por el Comité de Higiene de la SDN en 1935.

\section{LOS ENFOQUES INTERNACIONALES: LA ENCUESTA DE 1935}

La encuesta que llevaron a cabo en 1935 los médicos de la SDN, Dragoni y Burnet (1938), nació de un plan de cooperación entre esta institución y el gobierno de Chile, y que tenía como objetivo central realizar una investigación sobre el estado de la alimentación de los habitantes del país. En esa época la SDN estaba empeñada en asistir a los países miembros en el conocimiento sobre el estado nutricional de sus poblaciones, al igual que la OIT, aunque esta última estaba más interesada en estudiar el costo de vida y la capacidad de compra de la población trabajadora (Pernet, 2011). 
Los especialistas fueron apoyados por un comité constituido por la Dirección General de Sanidad, el cual debía formular un plan de trabajo (Dragoni y Burnet, 1938, p. 5). Se optó por aplicar la encuesta de manera descentralizada, y se asignó un papel relevante a los médicos jefes de los respectivos servicios públicos de las provincias, acompañados por enfermeras y visitadoras sociales, quienes debían entrevistar a las familias seleccionadas. Estas serían divididas en lo posible en grupos de quince familias, de acuerdo con su nivel de ingreso y actividad socioprofesional, y sus actividades cotidianas se seguirían durante dos semanas o al menos una.

El trabajo de campo se organizó en las siguientes etapas: a) preparación e impresión de los cuestionarios, $b$ ) redacción de los instructivos a los médicos provinciales y el personal técnico, $c$ ) determinación de los grupos socioprofesionales y sociales a los cuales se aplicaría la encuesta. Dragoni y Burnet, así como el comité asesor, eran conscientes de las particularidades geográficas del país, con una diversidad de climas y actividades productivas, por lo cual las conclusiones generales debían ser complementadas por el conocimiento sobre las decisiones que las familias tomaban en sus propias localidades. De esta forma se seleccionaron quince ciudades o localidades a lo largo del país, que comprendían 31 grupos de familias, con un alcance total de 593 familias y 3383 personas. La capital Santiago comprendió $41.3 \%$ del total de los encuestados, muy por encima de Valparaíso que alcanzó 8.7 por ciento.

El promedio de miembros por familia fue de 5.7, y se confirmó la idea de que las familias más pobres tenían un mayor número de hijos y otros miembros. De hecho, 35\% (1 182) de las personas encuestadas tenían entre cero y diez años de edad, y sólo $2.2 \%$ (75) eran personas calificadas como inactivas (mayores de 60 años de acuerdo con la ley) y de entre ellas 1.4\% (47) eran pensionados. En un desglose más detallado, predominan los lazos parentales padres-hijos, con $87.6 \%$, y a las relaciones no parentales, es decir allegadas y pensionistas, corresponde $4.7 \%$. Si bien esta última no era una cifra muy importante, sorprendió a los encuestadores que las familias más pobres tuvieran la costumbre de acoger en su seno a personas allegadas o pensionistas, las que colaboraban muy poco con el presupuesto familiar (Dragoni y Burnet, 1938, p. 23). En 58 familias, de un total de 593, las mujeres eran jefes de hogar.

Económicamente los jefes de familias dependían de actividades muy variadas, que incluían pescadores, vendedores ambulantes, costureras, trabajadores salitreros y marítimos, pintores, electricistas, obreros textiles y municipales, junto a "una aristocracia obrera", a decir de los autores, que correspondía a trabajadores de la industria del gas y de ferrocarriles, cuyos ingresos oscilaban entre menos de un peso hasta cinco y más por día, sien- 
do clasificados en seis grupos: menos de un peso; de 1.00 a 1.99; de 2.00 a 2.99 ; de 3.00 a 3.99 ; de 4.00 a 4.99 ; y 5.00 pesos y más.

Los resultados de la encuesta fueron consistentes con los otros estudios que hemos citado anteriormente, en especial en la relación existente entre consumo de calorías e ingreso económico. La investigación de Dragoni y Burnet estableció un promedio de consumo de 2357 calorías, de las cuales la menor ingesta correspondía al primer grupo (979 calorías), que ganaba menos de un peso por día, y la mayor (3 543 calorías), al grupo que ganaba alrededor de cinco pesos (véase cuadro 6). ${ }^{11} \mathrm{El}$ ingreso de entre 3.00 y 3.99 pesos (grupo 4) correspondía al punto de diferenciación entre subalimentación y alimentación, es decir, aproximadamente 2500 calorías, consideradas como una ración básica. Del total de 593 familias, casi $60 \%$ (349) no superaba las 2000 calorías, y sólo 19\% (114) consumía más de 3 000. ¿Qué explica esto?

Dragoni y Burnet (1938), apoyándose en una serie de anotaciones de los encuestadores, avanzaron en razones de orden material y cultural para explicar las deficiencias alimentarias de la población, aunque intentando no extraer conclusiones definitivas. El primer factor era el insuficiente nivel de ingresos que tenían las familias, cuyo $60 \%$ tenía un déficit presupuestario crónico, y estaban obligadas a endeudarse vía préstamos o crédito informal (p. 65). Los ingresos, además de reducidos, eran irregulares, tendencia común en todo el país, con la sola excepción de la localidad de Chacabuco, que era un yacimiento de salitre. Los autores también hicieron referencia a factores culturales, como la desorganización familiar y el desconocimiento de principios económicos básicos. Los jefes de familias se mostraban reacios a informar a sus esposas cuánto ganaban, y se calcula que cerca de $43 \%$ de los trabajadores entregaba al hogar menos dinero de lo que recibía (p. 40). Lo retenido se calculaba en 23\%, destinado a financiar gastos personales (aseo o vestimenta), ahorro para cubrir imprevistos y, en especial, consumo de alcohol y juego. De esta forma, gran parte del esfuerzo familiar recaía en las esposas y los hijos, quienes debían complementar el ingreso del esposo. Esto se tradujo en una alta tasa de inasistencia escolar, pues $54 \%$ de los niños de las familias que tenían ingresos menores a un peso diario no iba al colegio, a diferencia de las familias que ganaban unos tres pesos, y cuyo porcentaje de inasistencia escolar se reducía a 27\% (p. 73). En síntesis, esta encuesta, en especial por su metodología y alcance, no sólo corroboró el serio déficit nutricional que habían advertido los estudios anteriores, sino que además colocó en el centro de

\footnotetext{
${ }^{11}$ Este ingreso se calculó a partir de la cantidad de personas que trabajaban y el número total que ese ingreso debía mantener. En esta lógica, mientras más personas trabajan en una familia y menos son las cargas, mejor es su estándar de vida.
} 
CUADRO 6. CALORÍAS POR GRUPO SOCIOECONÓMICO

\begin{tabular}{lccr}
\hline Grupo & Ingreso (pesos) & Familias & Calorías \\
Grupo 1 & Menos 1.00 & 17 & \\
Grupo 2 & $1.00-1.99$ & 171 & 179 \\
Grupo 3 & $2.00-2.99$ & 161 & 1954 \\
Grupo 4 & $3.00-3.99$ & 130 & 2577 \\
Grupo 5 & $4.00-4.99$ & 66 & 3270 \\
Grupo 6 & 5.00 y más & 48 & 3543 \\
\hline
\end{tabular}

Fuente: elaboración propia con base en Dragoni y Burnet (1938).

la discusión las condiciones sociales y económicas que presentaban las familias chilenas en el contexto de la gran depresión.

\section{CONSIDERACIONES FINALES}

Sin minimizar los posibles problemas metodológicos que mostraron algunas de las encuestas de nutrición aplicadas entre 1928 y 1938, hemos querido, mediante su puesta en valor, destacar la importancia que tuvo el ítem de la alimentación en el consumo de las familias de trabajadores durante la gran depresión. La canasta de productos que creó la DGE en 1928 para calcular el ICV, si bien fue fundamental en el desarrollo de una temprana institucionalidad microeconómica, minimizó la importancia real que tenían los alimentos en la estructura de gastos de las familias y su peso en la variación del costo de la vida.

La mayoría de las encuestas de nutrición que hemos descrito dan cuenta de una subalimentación crónica de las familias trabajadoras chilenas, en especial de las más pobres. Medido en términos de ingesta calórica, el promedio de consumo se situó cerca de las 2300 calorías, por debajo de los requerimientos necesarios para realizar un esfuerzo mediano. También las encuestas revelan las profundas diferencias existentes entre el consumo de calorías de trabajadores vinculados a actividades mineras, industriales y agrícolas. Los estudios basados en los censos agropecuarios y demográficos son interesantes porque muestran tendencias que vienen a corroborar o matizar las encuestas de la década de 1930, y en especial porque sugieren como posible causa de los problemas nutricionales las condiciones de producción y distribución de los alimentos. En particular, las encuestas analizadas permiten concluir las principales deficiencias de los trastornos 
alimenticios: escasez en el consumo de los llamados alimentos protectores, como verduras, frutas y lácteos; poco consumo de proteínas de origen animal, como carne y huevo, y una dieta alta en contenido de cereales, en especial de pan. Para la comunidad de médicos la mala alimentación era la causa directa de las pésimas estadísticas vitales que presentaba el país durante el periodo de estudio, con una de las tasas más altas de mortalidad infantil en el mundo $(250 \%)$ y con una esperanza de vida que no superaba los 40 años en hombres y 43 años en mujeres (Instituto Nacional de Estadística, 2010).

Las encuestas, en especial la de Dragoni y Burnet (1938), muestran patrones de conformación familiar característicos de sociedades pobres y desiguales, donde las políticas sociales están en plena instauración. Si para el discurso oficial la familia era el centro donde se organizaban los presupuestos, se construían los gustos y se tomaban las decisiones de compra, debía ser intervenida por medio de políticas educativas y sanitarias con el fin de mejorar la administración de sus ingresos, incluso si estos eran escasos. En este punto coincidían algunos diagnósticos que responsabilizaban a la educación de los trabajadores y a su moral por la mala alimentación de las familias.

Hemos destacado la encuesta de Dragoni y Burnet porque forma parte de un esfuerzo de política internacional en materia de conocimiento sobre el estado nutricional de los países del continente latinoamericano. Si bien no estamos en condiciones de ponderar exactamente la influencia de esta encuesta en las mejoras metodológicas para medir la ingesta de calorías de la población chilena, sí podemos señalar que el solo hecho de su aplicación supuso un esfuerzo inédito de parte de diversos estamentos públicos y profesionales, junto a la participación de una temprana burocracia social interesada en los problemas de los trabajadores (visitadoras sociales). En este sentido, las encuestas del periodo, y en especial las discusiones que suscitaron, ayudaron a convertir a los médicos en agentes clave para abordar el problema alimenticio y proponer posibles soluciones, creando un marco ideológico y científico propicio a la intervención sanitaria. La experiencia inédita de la encuesta de la SDN de 1935 contribuyó a conectar a los profesionales chilenos con enfoques nuevos que se manejaban en las instituciones internacionales, lo que ayudó a crear una primera institucionalidad en materia de políticas alimentarias.

La creación del Consejo Nacional de Alimentación (CNA) en febrero de 1937 significó, en el ámbito institucional, la consagración de la alimentación como dominio de política pública. ${ }^{12}$ En su seno se reunió la comunidad médica interesada en promover acciones que enfrentaran los

${ }^{12}$ Decreto núm. 80, Diario Oficial, núm. 17.699, 20 de febrero de 1937. 
problemas nutricionales de la población de escasos recursos y en especial de mujeres y niños. Con la dirección del ministro de Salud Eduardo CruzCoke, y secundado por médicos y funcionarios públicos del más amplio espectro, el CNA se encargó de realizar estudios y proponer medidas legislativas sobre la materia. Entre las actividades asociadas al CNA podemos nombrar la difusión de cartillas de alimentación, restaurantes populares, estudios nutricionales, campañas de promoción de la leche, entre muchas otras medidas, que tuvieron su consagración con la aprobación en 1937 de la Ley Madre-Niño, que se ocupó del binomio considerado clave en la reducción de la desnutrición y mortalidad infantil, y en 1938 la promulgación de la Ley de Medicina Preventiva.

\section{LISTA DE REFERENCIAS}

Bayardo, L. (2013). Historia del consumo moderno en la ciudad de México durante los años 1909-1970 a través de las encuestas de gastos familiares y de la publicidad en la prensa (Tesis doctoral). El Colegio de México, México.

Bourges, H., BengoA, J. M. y O'Donnell, A. M. (coords.) (2002). Historias de la nutrición en América Latina. Buenos Aires: Sociedad Latinoamericana de Nutrición. Recuperado de http://www.sochinut.cl/pdf/HistNut_SLAN.pdf

Braun, J., Braun, M., Briones, I., Díaz, J., Lüders, R. y Wagner, G. (2000). Economía chilena, 1810-1995: Estadísticas históricas. Santiago de Chile: Instituto de Economía, Universidad Católica.

Carpenter, K. J. (1994). Protein and energy: a study of changing ideas in nutrition, Cambridge: Cambridge University Press.

Carreras, A., Hofman, A., Tafunell, X. y YáÑez, C. (2003). El desarrollo económico de América Latina en épocas de globalización. Una agenda de investigación. Serie Estudios Estadísticos y Prospectivos, 4. Santiago de Chile: Comisión Económica para América Latina y el Caribe.

CRUZ-COKE, E. (junio, 1928). Los equilibrios alimenticios y la alimentación del pueblo chileno (clase inaugural del curso de Química-Fisiológica y Patológica de 1928). Revista Médica de Chile, 4, 319-348.

Cueto, M. (2000). El regreso de las epidemias. Salud y sociedad en el Perú del siglo XX. Lima: Instituto de Estudios Peruanos.

CufF, T. (2004). The hidden cost of economic development: The biological standard of living in Antebellum Pennsylvania. Reino Unido: Ashgate Publishing Limited.

Dagnino, V. (enero-agosto, 1928). Causas de disolución de la familia y mortalidad infantil. Revista de la Sociedad Médica de Valparaíso, 10, 696-703.

Debré, R. (1930). Les enquêtes entreprises en Amérique du Sud sur la mortalité infantile. Ginebra: Service des Publications de la Société des Nations. 
DÍAZ, J., LÜDERS, R. y WAGNER, G. (1998). Economía chilena 1810-1995: evolución cuantitativa del producto total y sectorial (núm. 186). Santiago de Chile: Pontificia Universidad Católica de Chile.

Diego, M. P., Peña, R. L. y Peralta, C. C. (2002). La Asamblea Obrera de Alimentación Nacional: un hito en la historia de Chile. Chile: Sociedad Chilena de Sociología.

Dirección General de Estadística [DGE] (febrero, 1928a). Estadística Chilena, 2, 22-46.

Dirección General de Estadística [DGE] (noviembre, 1928b). Estadística Chilena, $11,350-355$.

Dirección General de Estadística [DGe] (diciembre, 1928c). Estadística Chilena, 12. Dirección General de Estadística [DGE] (marzo, 1929). Estadística Chilena, 3, 100-120.

Dirección General de Estadística [DGE] (1930). Anuario Estadístico de la República de Chile, año 1928. Santiago de Chile: Autor.

Dragoni, C. y Burnet, E. (1938). L'alimentation populaire au Chili. Santiago de Chile: Imprenta Universo.

Federico, G. (2005). Feeding the world: An economic history of agriculture, 1800-2000. Nueva Jersey: Princeton University Press.

FogEL, R. (1993). New sources and new techniques for the study of secular trends in nutritional status, health, mortality, and the process of aging. Historical Methods, 26(1), 1-44.

FogEL, R. (2009). Escapar del hambre y de la muerte prematura. 1700-2100. Madrid: Alianza Editorial.

Garrido, S. (2014). Niveles de vida y conflictividad laboral en los obreros de la Gran Minería del Cobre, 1911-1991 (Tesis de maestría). Universidad de Chile, Santiago de Chile.

GonzÁlez, R. (1935). Cómo se alimenta la familia obrera en Santiago. Revista de Medicina y Alimentación, 1, 15-25.

Hargrove, J. L. (2006). History of the calorie in nutrition. The Journal of Nutrition, 12(136), 2957-2961.

Illanes, M. A. (2006). Cuerpo y sangre de la política. La construcción histórica de las visitadoras sociales (1887-1940). Santiago de Chile: Lom Ediciones.

Instituto NACIONAL DE EsTADÍsTICA (1957). Índice de precios al consumidor. Índice de costo de vida. Santiago de Chile: Autor, 2007.

Instituto Nacional de Estadística (2010). Hombres y mujeres en Chile. Santiago de Chile: Autor, 2010.

KLubock, T. (1998). Class, gender, and politics in Chile's El teniente Copper Mine, 19041951. Durham: Duke University Press.

Lipschutz, A. (octubre, 1933). La ciencia de la alimentación. Revista de Medicina y Alimentación, 1, 3-8.

Lluch, A. (2015). Las manos visibles del mercado. Intermediarios y consumidores en la Argentina. Rosario: Protohistoria Ediciones.

LóPez-Alonso, M. (2015). Estar a la altura. Una historia de los niveles de vida en México, 1850-1950. México: Fondo de Cultura Económica. 
Marchand, C. (2014). Le médecin et l'alimentation. Principes de nutrition et recommandations alimentaires en France (1887-1940) (Tesis doctoral). Université François-Rabelais, Tours.

Mardones, J. (enero, 1935). El problema de la alimentación en Chile. Revista de Medicina y Alimentación, 6, 367-378.

Matus, M. (2012). Crecimiento sin desarrollo. Precios y salarios reales durante el ciclo salitrero en Chile (1880-1930). Santiago de Chile: Editorial Universitaria.

Milanesio, N. (2014). Cuando los trabajadores salieron de compras. Nuevos consumidores, publicidad y cambio cultural durante el primer peronismo. Buenos Aires: Siglo XXI Editores.

Ministerio de SAlud (2010). Nutrición para el desarrollo. Claves del éxito del modelo chileno. Santiago de Chile: Autor.

Oficina del Trabajo (primer trimestre, 1911). Boletín de la Oficina del Trabajo, 1(1), 90-101.

Oficina del Trabajo (primer trimestre, 1912). Boletín de la Oficina del Trabajo, 1(1), 60-92.

Organización Internacional del Trabajo (1936). Conferencia del trabajo de los Estados de América miembros de la Organización Internacional del Trabajo. Ginebra: Autor.

PÉrez, I. (segundo semestre, 2015). Apuntes para el estudio del consumo en clave histórica. Avances del Centro de Estudios Sociales Regionales, 13(12), 97-106.

Pernet, C. (2011). L'oit et la question de l'alimentation en Amérique latine (19301950). Les problèmes posés par la définition internationale des normes de niveau de vie. En V. Viet y I. LeSPINET-Moret, L'Organisation internationale du travail (pp. 167-177). Rennes: Presses Universitaires de Rennes.

Popkin, B. M. (marzo, 1993). Nutritional patterns and transitions. Population and Development Review, 1(19), 138-157.

Popkin, B. M. y Doak, C. M. (1998). La epidemia de obesidad es un fenómeno mundial. Nutrition Reviews, 56(4), 106-114.

REyes, N. (2015). Salarios agrícolas durante la industrialización en Chile: factores económicos e institucionales. Estudios de Economía, 42(2), 121-141.

REYES, R. (1933). Encuesta de nutrición a partir de censos agropecuarios y de población (Tesis). Universidad de Chile, Santiago de Chile.

Rinke, S. (2014). Encuentros con el yanqui: norteamericanización y cambio sociocultural en Chile (1898-1990). Santiago de Chile: Dirección de Bibliotecas, Archivos y Museos.

RodAs, Á. (enero, 1937). Algunos aspectos de la alimentación popular en Chillán. Revista de Medicina y Alimentación, 6, 271-276.

Rodríguez, J. (junio, 2013). Economía política de la distribución del ingreso rural en Chile durante la decadencia de la Hacienda, 1935-1971. Revista Uruguaya de Historia Económica, 3(3), 33-62.

Rodríguez, J. (2014). La economía política de la desigualdad de ingreso en Chile, 1850-2009 (Tesis doctoral). Universidad de la República, Uruguay. 
Rosemblatt, K. (1995). Masculinidad y trabajo: el salario familiar y el Estado de compromiso, 1930-1950. Proposiciones, 26, 70-86.

Rosemblatt, K. (2000). Gendered compromises: political cultures and the state in Chile, 1920-1950. Chapel Hill: University of North Carolina Press.

SAIKI, T. (1928). Necesidad de estudiar la nutrición. Revista de la Sociedad Médica de Valparaíso, 8, 507-515.

Salas, D. (1932). Cartilla de alimentación. Santiago de Chile: Ediciones Restaurant Naturista.

Santa María, J. (1933). Contribución al estudio de la alimentación popular (Extracto de memoria para optar al grado de médico cirujano). Universidad de Chile, Santiago de Chile.

Santa María, J. (1935). La alimentación de nuestro pueblo. Santiago de Chile: Talleres de San Vicente.

SANTA MARÍA, J. (enero-abril, 1949). Características de nuestros hábitos alimentarios. Revista de Medicina y Alimentación, 4-5-6, 116-125.

Seibert, S. (1936). Legislación del trabajo y previsión social en "El Teniente" (Tesis de licenciatura). Universidad de Chile, Santiago de Chile.

SMIL, V. (2000). Feeding the world. A challenge for the twenty-first century. Cambridge: Massachusetts Institute of Technology Press, 2000.

SteCKel, R. y Rose, J. (2002). The backbone of history: health and nutrition in the western hemisphere, Cambridge: Cambridge University Press.

Tablas de alimentación recomendadas por la Comisión Técnica de Nutrición de la Comisión de Higiene de la Sociedad de Naciones (agosto, 1938). Boletín del Ministerio de Salubridad, 8, 30-31.

Thompson, E. P. (1979). Tradición, revuelta y conciencia de clase. Barcelona: Crítica.

Torre, J. C. y PAstorizA, E. (2002). La democratización del bienestar. En J. C. Torre (dir.), Los años peronistas (1943-1955). Nueva Historia Argentina, t. VII (pp. 257-312). Buenos Aires: Sudamericana.

TORRes, I. (1938). Alimentación de las clases populares (Tesis de licenciatura). Universidad de Chile, Santiago de Chile.

VArgas, J. (2011). Alimentar el cuerpo social: ciencia, dieta y control en México durante el porfiriato (Tesis de maestría). Universidad Autónoma de México, México.

Vargas, N. (2002). Historia de la pediatría chilena: crónica de una alegría. Santiago de Chile: Editorial Universitaria.

VergarA, Á. (2008). Copper workers, international business, and domestic politics in Cold War Chile. Pennsylvania: The Pennsylvania University Press.

YÁÑEz, J. C. (2008). La intervención social en Chile (1907-1932). Santiago de Chile: Ril Editores.

ZÁrate, M. S. (2012). Alimentación y previsión biológica: la política médico-asistencial de Eduardo Cruz-Coke (Estudio introductorio). En C. CRuz-Coke, Medicina preventiva y medicina dirigida. Santiago de Chile: Cámara Chilena de la Construcción/ Pontificia Universidad Católica de Chile/Biblioteca Nacional. 
ZÁrATE, M. S. (2013). El licor de la vida. Lactancia y alimentación materno-infantil, Chile (1900-1950). En C. Sciolla (comp.), Historia y cultura de la alimentación en Chile: miradas y saberes sobre nuestra culinaria. Santiago de Chile: Catalonia.

\section{OtRAS FUENTES}

Diario Oficial, Santiago, Chile. 